\title{
Caractérisation d'une collection de souches de bactéries corynéformes de la morge du gruyère de Comté
}

\author{
C Piton, C Fontanier
}

INRA, Station de recherches en technologie et analyses laitières, BP 89, 39800 Poligny, France

(Reçu le 21 mai 1990; accepté le 2 juillet 1990)

\begin{abstract}
Résumé - Cent dix huit souches de bactéries corynéformes isolées de la morge du Comté dans 6 fromageries et à 2 stades de l'affinage des fromages ont été caractérisées sur la base de 68 tests biochimiques et physiologiques. Les résultats obtenus sur 59 souches orange opaques d'une part, et sur 59 souches non pigmentées d'autre part, ont ensuite été soumis à une analyse numérique sur la base de l'indice de similitude de Jaccard et du critère du saut minimal. Avec $80 \%$ de similitude entre elles, 54 des 59 souches orange opaques forment un seul groupe homogène. Les 59 souches non pigmentées ont été divisées en 4 classes principales : toutes les colonies opaques sont regroupées dans deux d'entre elles (Classes I et IV) et toutes les colonies translucides dans les deux autres (Classes II et III). Les classes de souches orange et de souches non pigmentées ont ensuite pu être divisées en différentes sous-classes et on a sélectionné des critères qui permettent d'identifier les souches appartenant à ces différentes classes et sous-classes. Aucun des groupes de souches ne semble spécifique d'une fromagerie mais quelques-uns d'entre eux ont été isolés plus souvent au début et d'autres en fin d'affinage des fromages.
\end{abstract}

\section{Comté / morge / bactérie corynéformes / analyse numérique}

Summary - Characterization of coryneform bacteria isolated from the Comté cheese smear. One hundred and eighteen strains of coryneform bacteria isolated from the Comté cheese smear in 6 plants and at 2 stages of ripening have been characterized using 68 biochemical and physiological tests. The results obtained for the 59 opaque orange strains and for the 59 non pigmented strains were submitted to numerical analysis, using the Jaccard's similarity coefficient. With a similarity index of $80 \%, 54$ of the 59 orange strains formed a slightly homogenous cluster. The 59 non pigmented isolates were divided into 4 main clusters: the opaque colonies were grouped into two of them (Clusters I and IV) and the translucent colonies in the other two (Clusters II and III). The clusters of orange and non pigmented isolates were then divided into different subclusters and criteria were selected, which allowed the identification of strains belonging to the different clusters or subclusters. No group of strains seems to be specific to the cheese plant but some of them have been isolated preferably at the beginning and others at the end of the cheese ripening period.

Comté cheese / smear / coryneform bacterium / numerical analysis 


\section{INTRODUCTION}

Dans une précédente étude (Piton, 1988), nous avons montré qu'une flore microbienne s'implante très rapidement à la surface du Gruyère de Comté et atteint des niveaux très élevés, avec une succession levures / bactéries; après 15 jours d'affinage, la flore bactérienne domine et se stabilise à son niveau maximum (environ $10^{10}$ micro-organismes $/ \mathrm{cm}^{2}$ de croûte). Cette flore bactérienne de la morge est halotolérante et se compose essentiellement de bactéries corynéformes réparties en trois grands groupes pigmentaires qui se maintiennent à un niveau constant, tout au long de l'affinage et quelle que soit la fromagerie.

Contrairement à ce qui a été observé pour d'autres types de fromages (Lenoir, 1963; Richard et Zadi, 1983; Seiler, 1986), les concentrations de cette flore corynéforme à la surface du Comté semblent peu varier en fonction de l'origine des fromages; de même, les proportions entre les groupes pigmentaires semblent stables. Cela peut signifier que cette flore est assez peu dépendante de la technologie mise en œuvre (température, hygrométrie, fréquence et qualité des soins en cave, qualité de l'ensemencement naturel des meules). Cependant, les propriétés physiologiques et/ou biochimiques des microorganismes appartenant à chaque groupe pigmentaire sont peut-être très différentes d'une fromagerie à l'autre ou d'une cave d'affinage à l'autre. De même, un changement de la nature des bactéries corynéformes a pu se produire au cours de l'affinage, comme cela a déjà été observé pour de nombreux groupes bactériens dans les fromages (Lenoir et Auberger, 1966; Kikuchi, 1966; Mourgues et al, 1977; Richard, 1984). L'apparente stabilité des courbes observées résulterait alors de la superposition de plusieurs courbes en cloche (croissance et déclin plus ou moins accen- tués) des différents types bactériens constituant cette flore halotolérante.

II nous a donc semblé intéressant de soumettre une collection de souches de bactéries corynéformes, représentative de la flore dominante de la morge du Comté, à une série de tests physiologiques et biochimiques. Les résultats à ces différents tests devraient nous permettre de classer les souches dominantes en différents types en fonction de leurs caractéristiques et de révéler leur variabilité. Enfin, cela devrait nous conduire à retenir une batterie de tests simples permettant l'identification de nouvelles souches aux différents types bactériens mis en évidence; cet outil est indispensable à une étude plus large de la flore bactérienne de la morge du Comté et d'autres fromages (Beaufort, par exemple) : variabilité de cette flore en fonction du type d'affinage et évolution des différents types bactériens au cours de l'affinage.

\section{MATÉRIEL ET MÉTHODES}

\section{Origine des souches}

Cent dix-huit souches de bactéries corynéformes isolées de la morge du Gruyère de Comté ont été prises au hasard dans la collection obtenue à partir de six fromageries lors d'une étude précédente (Piton, 1988). Quarante sept d'entre elles ont été prélevées en début d'affinage des fromages ( 8 jours après fabrication), 71 en fin d'affinage (60 et 90 jours après fabrication). Cinquante neuf d'entre elles donnent des colonies pigmentées orange opaques produisant une teinte rouge au contact d'une solution de soude $5 \mathrm{~N}$ (Jones et al, 1973); les 59 autres donnent des colonies non pigmentées.

\section{Caractérisation}

Les 118 souches ont été soumises à 68 tests biochimiques et physiologiques. Ces tests ont 
été choisis pour trois raisons principales : ils sont faciles à mettre en œuvre sur un grand nombre de souches; ces critères phénotypiques ont déjà permis à d'autres auteurs de réaliser un classement des corynébactéries en divers groupes (Yamada et Komagata, 1972; Crombach, 1974b; Jones, 1975; Zadi, 1981; Seiler, 1983 et 1986); ils peuvent présenter un intérêt écologique (croissance à différents $\mathrm{pH}$ ou températures) ou technologique, comme la production de composés d'arômes liée à la dégradation de certains acides aminés (Hemme et al, 1982; Hemme et Richard, 1986).

\section{Utilisation de différents substrats comme seule source de carbone et d'énergie}

Nous avons utilisé comme substrats les 46 composés suivants : 7 acides gras (Prolabo) (acétate, propionate, butyrate, caproate, caprylate, pélargonate et caprate); 6 acides dicarboxyliques (succinate (Prolabo), pimélate, azélate et sébacate (Sigma), malonate et fumarate (Merck); 2 hydroxyacides (DL-lactate (Prolabo), DL-3-hydroxybutyrate (Sigma); 5 autres acides organiques (citrate (Prolabo), $\alpha$-cétoglutarate, pyruvate, itaconate et mésaconate (Sigma); 3 composés cycliques (Sigma) (m-hydroxybenzoate, $p$-hydroxybenzoate et phénylacétate); 17 acides aminés aliphatiques (glycine (Prolabo), L- $\alpha$-alanine, $\quad \mathrm{D}-\alpha$-alanine, $\quad \mathrm{B}$-alanine, Lsérine, L-thréonine, L-leucine, L-isoleucine, DLnorleucine, L-valine, L-aspartate, L-glutamate, Llysine, L-arginine, D-ornithine, $\delta$-aminovalérate et L-méthionine (Sigma); 5 acides aminés contenant un noyau benzènique (Sigma) (L-histidine, L-proline, L-tyrosine, L-phénylalanine et Ltryplophane); 1 autre composé azoté (hippurate (Difco)).

Ces milieux sont préparés par addition du substrat au milieu minéral de composition suivante (Stanier et al, 1966): $\mathrm{MgSO}_{4}, 7 \mathrm{H}_{2} \mathrm{O}, 0,2$ g; $\mathrm{K}_{2} \mathrm{HPO}_{4}, 1 \mathrm{~g} ; \mathrm{FeSO}_{4}, 7 \mathrm{H}_{2} \mathrm{O}, 0,05 \mathrm{~g} ; \mathrm{CaCl}_{2}$, $0,02 \mathrm{~g} ; \mathrm{MnCl}_{2}, 4 \mathrm{H}_{2} \mathrm{O}, 0,002 \mathrm{~g} ; \mathrm{NaMoO}_{4}, 2 \mathrm{H}_{2} \mathrm{O}$, $0,001 \mathrm{~g} ; \mathrm{NH}_{4} \mathrm{Cl}, 1 \mathrm{~g}$; gélose (Pastagar, Institut Pasteur Production), $13 \mathrm{~g}$; eau distillée, $1 \mathrm{I}$. Le $\mathrm{pH}$ du milieu est ajusté à 7,0 à l'aide de $\mathrm{NaOH}$ ou $\mathrm{HCl}$ concentré. Après stérilisation, on ajoute à ce milieu $2,5 \mathrm{ml}$ par litre d'une solution de vitamines, stérilisée par filtration, de la composition suivante (Bousfield, 1972) : acide $p$ aminobenzoïque, $2 \mathrm{mg}$; acide folique, $2 \mathrm{mg}$; bio- tine, 0,4 mg; acide nicotinique, $40 \mathrm{mg}$; thiamine, 40 mg; vitamine B12, 0,2 mg; acide pantothénique, $40 \mathrm{mg}$; eau distillée, $100 \mathrm{ml}$.

Les acides organiques sont ajoutés au milieu à la concentration de $0,1 \%$, comme le préconisent Stanier et al (1966), les acides aminés à la concentration de $0,4 \%$ (Zadi, 1981).

Tous les composés solubles dans l'eau sont stérilisés à part, par filtration sur filtre de porosité $0,22 \mu \mathrm{m}$. Les acides aminés et organiques insolubles dans l'eau sont préalablement solubilisés par diminution ou augmentation du $\mathrm{pH}$.

Ces différents substrats sont ajoutés au milieu minéral de base, vitaminé au moment de l'emploi; le pH est alors ajustée à 7,0 .

\section{Résistance aux antibiotiques}

Nous avons étudié les résistances à la pénicilline G (4 unités/100 ml), la bacitracine (10 unités $/ 100 \mathrm{ml})$, la carbénicilline $(100 \mu \mathrm{g} / 100 \mathrm{ml})$, la gentamycine $(20 \mu \mathrm{g} / 100 \mathrm{ml})$, la rifamycine (10 $\mu \mathrm{g} / 100 \mathrm{ml})$, l'acide nalidixique $(4 \mathrm{mg} / 100 \mathrm{ml})$, la sulfadiazine $(600 \mu \mathrm{g} / 100 \mathrm{ml})$, la streptomycine $(20 \mu \mathrm{g} / 100 \mathrm{ml})$ et la terramycine $(60 \mu \mathrm{g} / 100 \mathrm{ml})$.

Les concentrations finales citées sont celles employées par Zadi (1981). Les antibiotiques sont extraits de disques (Difco ou Institut Pasteur) par trempage dans l'eau distillée stérile pendant $1 \mathrm{~h}$, La solution obtenue est mélangée, juste avant emploi, au milieu Mueller Hinton (MH, Institut Pasteur Production).

\section{Résistance au $\mathrm{NaCl}$}

Nous avons utilisé la gélose nutritive (Lenoir, 1963) additionnée de $12,5,15,0,17,5$ ou $20,0 \%$ de $\mathrm{NaCl}$.

\section{Croissance à différents pH}

Quatre valeurs de $\mathrm{pH}$ ont été testées sur gélose nutritive (Lenoir, 1963) : 5,5, 6,0,9,5 et 10,0. Ces pH ont été obtenus par addition de $\mathrm{NaOH}$ ou de $\mathrm{HCl}$ auparavant stérilisés par filtration.

\section{Croissance à différentes températures}

Nous avons testé les températures de 4, 7, 12 et $37^{\circ} \mathrm{C}$ sur gélose nutritive. Pour les tempéra- 
tures de 4,7 et $12^{\circ} \mathrm{C}$, les lectures ont été effectuées à la chambre froide pour éviter une augmentation de la température des boîtes de Petri qui pourrait entraîner des résultats faux positifs.

\section{Pigmentation des colonies}

La pigmentation des colonies est mise en évidence sur milieu MSA après 3 jours d'incubation à $30^{\circ} \mathrm{C}$, puis 2 à 3 semaines à température ambiante et à la lumière.

\section{Ensemencement des milieux}

Les colonies microbiennes sont d'abord prélevées sur milieu MSA (cultures âgées d'environ 10 jours) et mises en suspension dans $1,5 \mathrm{ml}$ de liquide de Ringer dilué au quart, jusqu'à obtention de la concentration bactérienne souhaitée (environ $10^{8}$ cellules $/ \mathrm{ml}$ ). Les tubes à essai sont ensuite agités au vortex pendant $15 \mathrm{~s}$ pour obtenir une répartition homogène des microorganismes, puis placés dans de la glace fondante jusqu'à l'ensemencement (au plus 30 $\min$ ).

Les suspensions microbiennes sont alors déposées dans les alvéoles d'une boîte de Petri à 25 compartiments (1 ml/alvéole) et transférées à l'aide d'un appareil de transfert automatique (Multipoint Inoculator, Grande Bretagne) sur les différents milieux répartis en boîtes de Petri à 25 compartiments. Après absorption de l'inoculum dans la gélose, les boîtes ensemencées sont retournées et mises à l'étuve à $28^{\circ} \mathrm{C}$ (sauf pour les cultures à diverses températures).

\section{Lecture et notation}

Six lectures sont effectuées par comparaison avec un milieu témoin (milieu sans substrat, sans $\mathrm{NaCl}$ ou sans antibiotique) : après $3,4,6$, 9,13 et 17 jours d'incubation. Nous n'avons pas poursuivi les lectures au delà de 17 jours pour deux raisons : tendance au desséchement du milieu et absence de nouveaux résultats positifs. Dans chaque cas, ces lectures ont été faites par deux opérateurs.
Pour l'utilisation des acides aminés et des acides organiques, nous avons considéré comme positives les souches ayant crû plus nettement que sur le témoin; quant aux autres tests, nous avons considéré comme positives les souches dont la croissance était aussi abondante que sur le milieu témoin.

\section{Classification}

Les résultats de l'étude phénotypique ont fait l'objet d'une analyse numérique sur la base de 60 des 68 critères décrits précédemment. Nous avons éliminé de l'étude 7 tests pour lesquels les 118 souches présentent une réponse positive (croissance à pH 9,5; utilisation du lactate; résistance à la sulfadiazine, à la terramycine, à l'acide nalidixique et à la pénicilline) ou négative (utilisation du caprate); par ailleurs, nous avons réalisé une étude séparée des souches orange opaques (59 souches) et des souches non pigmentées ( 59 souches). La réponse à chaque test a été notée 1 pour un résultat nettement positif et 0 pour un résultat négatif ou douteux.

Les calculs ont été effectués sur ordinateur. La première étape consiste à calculer les pourcentages de similitude entre les souches prises deux à deux, soit un total de $n(n-1) / 2$ comparaisons pour $n$ souches. Plusieurs formules de calcul ont été proposées (Sokal et Sneath, 1963). Nous avons utilisé l'indice de Jaccard $S_{J}$ qui ne tient compte, dans les similitudes, que des résultats positifs :

$$
S_{J}=N_{(1,1)} \mathcal{J}\left(N_{(0,1)}+N_{(1,0)}+N_{(1,1)}\right)
$$

où $N_{(1,1)}$ est le nombre de tests positifs pour les deux souches comparées, $N_{(1,0)}$ et $N_{(0,1)}$ les nombres de tests divergents entre les deux souches.

A partir de ces pourcentages de similitude entre souches, une matrice des distances 1-S est établie. Les souches sont alors comparées, en fonction de cette distance, suivant le critère du saut minimal (Benzecri, 1975). Un dendogramme illustrant la relation entre les souches d'un groupe donné et entre les groupes est enfin élaboré. 
RÉSULTATS

\section{Souches orange opaques}

Sur le dendogramme de la figure 1 , sont notées en ordonnée les 59 souches de la collection, regroupées en fonction de leurs similitudes, et en abscisse les distances taxonomiques. Ces souches présentent un certain nombre de caractères communs puisque au moins $95 \%$ des souches sont capables de croître à $\mathrm{pH} 10$, en présence de $17,5 \%$ de sel mais pas de $20 \%$ de sel, et d'utiliser comme seule source de carbone et d'énergie, l'acétate, le propionate et le caproate. Elles ne constituent cependant pas un ensemble homogène puisqu'elles ne sont reliées qu'à la distance de 0,577 . Bien que l'on observe en fait un continuum, nous avons séparé les souches en deux groupes d'importance très inégale : la classe I (54 souches) et la classe II ( 3 souches), avec respectivement des niveaux d'agrégation interne de 0,273 et 0,571 . Le tableau I présente les principaux caractères permettant de différencier ces deux groupes de bactéries. La classe II est donc caractérisée par son incapacité à utiliser l'ensemble des acides aminés testés comme seule source de carbone et d'énergie; il en est de même pour les acides organiques, à l'exception de l'acétate, du propionate et du caproate.

A l'inverse des souches de la classe II, les souches de la classe I forment un phénon assez homogène. Elles sont caractérisées, pour au moins $95 \%$ des souches, par leur résistance à la carbénicilline et leur sensibilité à la bacitracine ainsi que par leur capacité à utiliser comme seul substrat, les 15 composés suivants : acétate, propionate, butyrate, caproate, succinate, sébacate, DL-3-hydroxybutyrate, $\alpha$ cétoglutarate, $\mathrm{L}-\alpha$-alanine, aspartate, glutamate, arginine, $\delta$-aminovalérate, histidine et phénylalanine. Nous avons cependant distingué 8 principaux types représentant environ les trois quarts des souches testées. Les dix critères présentés sur le tableau II nous permettent de différencier les souches de ces divers types.

Aucun des types mis en évidence n'est rencontré préférentiellement dans l'une ou

Tableau I. Caractères distinctifs des deux principales classes de bactéries corynéformes orange opaques isolées de la morge du Comté (Pourcentage de réponses positives).

Biochemical characteristics of the two main clusters of orange coloured coryneform bacteria isolated from the Comté cheese smear (Percentage of positive strains).
Test.
Classe I Classe II

Utilisation de :

. butyrate

- caprylate

- succinate

- fumarate

- azélate

- DL 3 hydroxybutyrate

. $\alpha$ cétoglutarate

- pyruvate

p-hydroxybenzoate

- L- $\alpha$-alanine

D- $\alpha$-alanine

- valine

- aspartate

- glutamate

- arginine

- ornithine

. $\delta$-aminovalérate

- histidine

- proline

- phénylalanine

98,1

88,9

98,1

90,7

90,7

98,1

98,1

85,2

85,2

96,3

87,0

92,6

96,3

96,3

100

94,4

100

98,1

94,4

100

0
0
0
0
0
0
0
0
0
0
0
0
0
0
0
0
0
0
0
0

Nombre de souches 


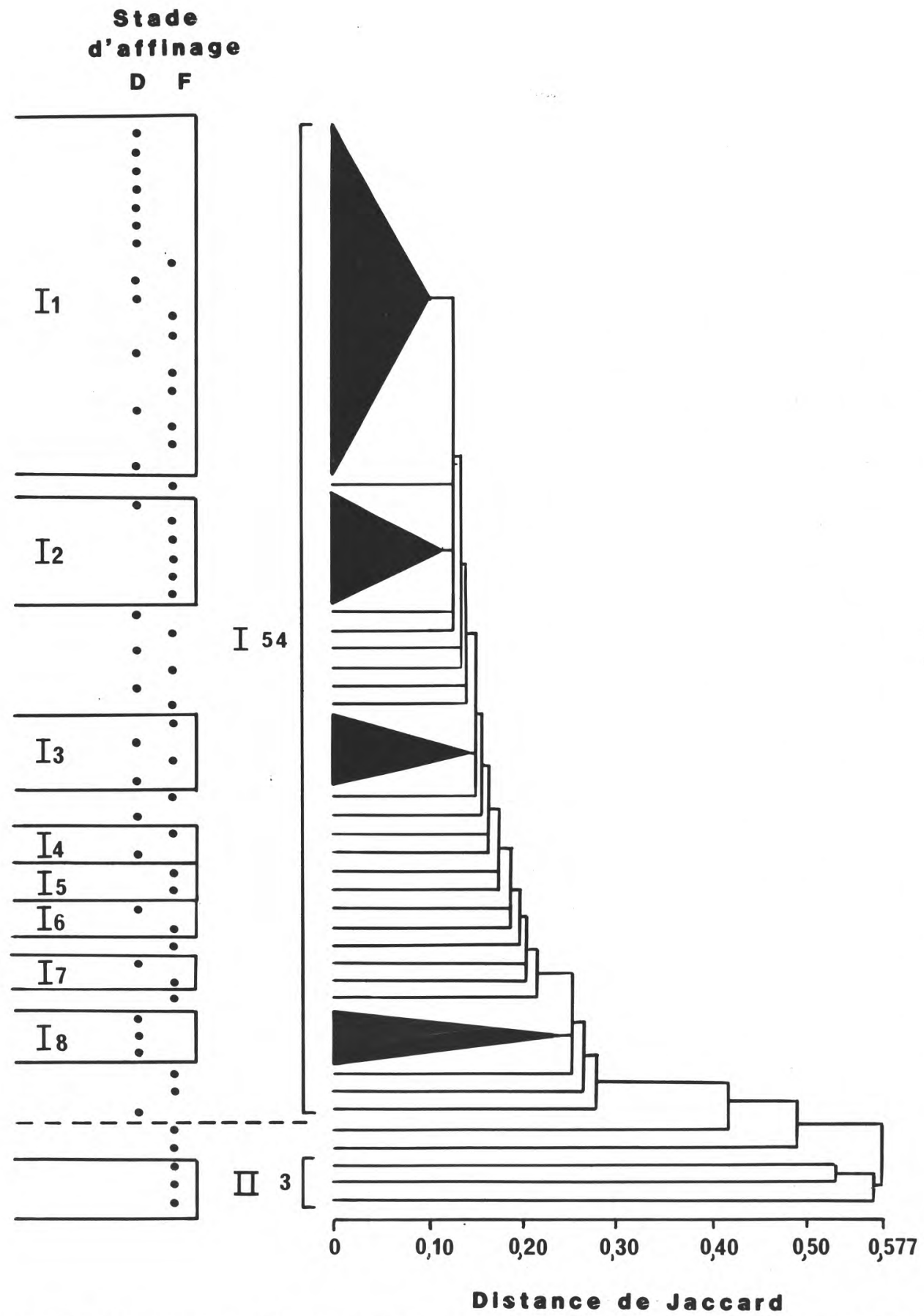

Fig 1. Dendrogramme simplifié de la classification, selon les distances de Jaccard, d'une collection de 59 souches orange opaques de bactéries corynéformes isolées de la morge du Comté. D : Début d'affinage; $F$ : Fin d'affinage.

Simplified dendrogram derived from the similarity matrix of the 59 opaque orange strains of coryneform bacteria isolated from the Comté cheese smear. $D$ : Beginning of ripening; $F$ : End of ripening. 
Tableau II. Caractères distinctifs des souches de bactéries corynéformes orange opaques appartenant aux 8 différentes sous-classes de la classe I (Pourcentage de réponses positives).

Characteristics of the orange coloured strains belonging to the 8 subclusters of the cluster I (Percentage of positive strains).

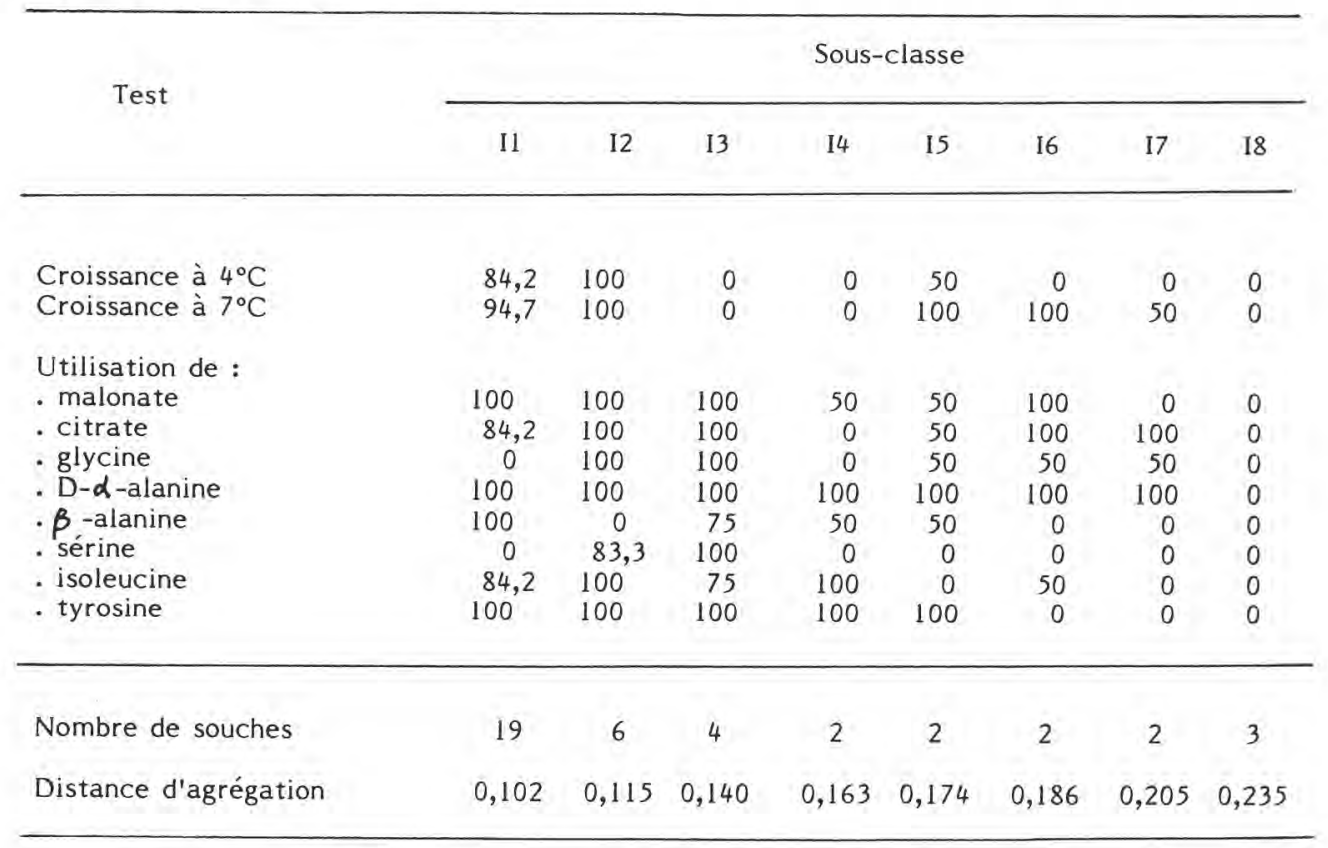

l'autre des fromageries de l'étude. Par contre, certains d'entre eux semblent être plus spécifiques d'un stade de l'affinage des fromages (fig 1): ainsi, les souches des types 11 et 18 semblent être isolées le plus souvent en début d'affinage ( 8 jours après la fabrication); celles des types 12 et II le plus souvent en fin d'affinage (60 et 90 jours après la fabrication). Cependant, compte tenu du faible nombre de souches étudiées, il n'est pas sûr que cette différence de répartition soit significative.

Par ailleurs, les souches de la classe I présentent en général des caractères nutritionnels analogues à celles isolées d'autres fromages par Seiler (1986) et identifiées à Brevibacterium linens (tableau III); ce n'est pas le cas des souches de la classe II.

\section{Souches non pigmentées}

La figure 2 montre sous forme d'un dendrogramme la relation entre les 59 souches non pigmentées testées. L'ensemble des souches constitue un groupe assez hétérogène puisqu'elles ne sont reliées qu'à la distance de 0,625 ; ainsi, elles présentent très peu de caractères communs : l'incapacité à croître à $37^{\circ} \mathrm{C}$ et en présence de $20 \%$ de sel et la capacité à croître à $\mathrm{pH} 10$. Nous avons constitué 4 groupes principaux de souches : les classes I (26 souches), II (18 souches), III (5 souches) et IV (5 souches), avec respectivement des distances d'agrégation interne de $0,237,0,333,0,348$ et 0,600 . L'aspect des colonies s'est révélé être un 


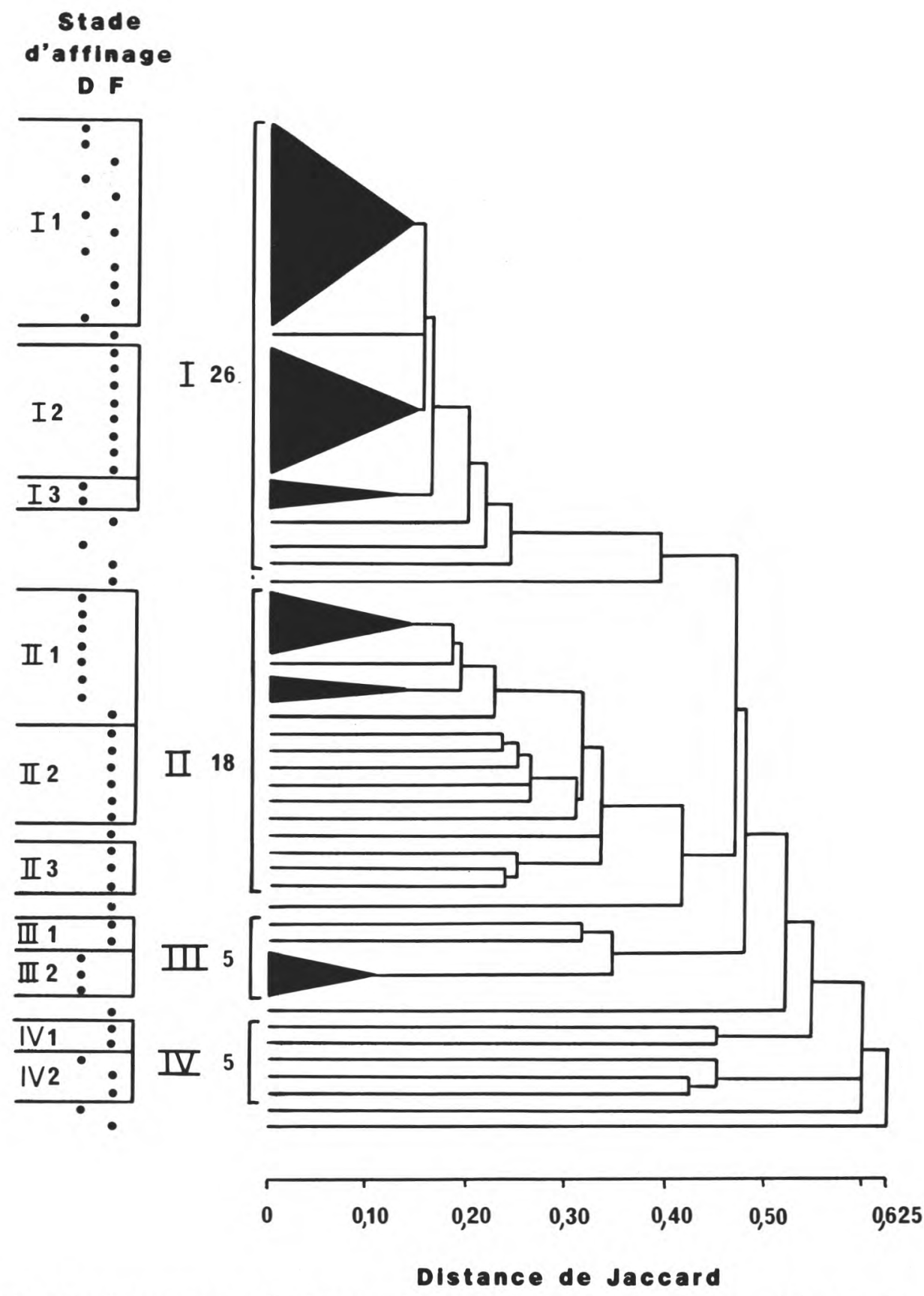

Fig 2. Dendrogramme simplifié de la classification, selon les distances de Jaccard, d'une collection de 59 souches non pigmentées de bactéries corynéformes isolées de la morge du Comté. D : Début d'affinage; $F$ : Fin d'affinage.

Simplified dendrogram derived from the similarity matrix of the 59 non pigmented strains of coryneform bacteria isolated from the Comté cheese smear. $D$ : Beginning of ripening; $F$ : End of ripening. 
Tableau III. Comparaison des caractères nutritionnels des souches de bactéries corynéformes orange opaques isolées de divers fromages et décrites par Seiler (1986) avec celles isolées de la morge du Comté (Pourcentage de réponses positives).

Comparison of biochemical characters of the orange coloured coryneform bacteria isolated by Seiler (1986) from various cheese varieties with those of the strains isolated from the Comté cheese smear (Percentage of positive strains).

\begin{tabular}{lccc}
\hline & Souches de & Souches de la morge du Comté \\
\cline { 3 - 4 } Substrat testé & & & \\
\cline { 3 - 4 } & SEILER (1986) & Classe I & Classe II \\
& & & \\
\hline & & & \\
Acétate & 95 & 100 & 40 \\
Propionate & 100 & 100 & 100 \\
Caprylate & 97 & 88,9 & 0 \\
S-aminovalérate & 90 & 100 & 0 \\
Malonate & 28 & 75,9 & 0 \\
Succinate & 91 & 98,1 & 0 \\
Citrate & 70 & 77,8 & 0 \\
Lactate & 100 & 100 & 100 \\
Fumarate & 89 & 90,7 & 0 \\
P-hydroxybenzoate & 99 & 85,2 & 0 \\
Glycine & 55 & 35,2 & 0 \\
Leucine & 99 & 83,3 & 0 \\
Proline & 99 & 94,4 & 0 \\
Thréonine & 40 & 40,7 & 0 \\
Aspartate & 80 & 96,3 & 0 \\
Histidine & 99 & 98,1 & 0 \\
Arginine & 97 & 100 & 0 \\
& & & \\
\hline Nombre de souches & 115 & 54 & 5 \\
& & & \\
\hline
\end{tabular}

critère intéressant de différenciation des souches : celles regroupées dans les classes I et IV donnent des colonies opaques, tandis que celles des classes II et III donnent des colonies translucides.

\section{Colonies opaques}

Les souches non pigmentées opaques de la classe I présentent des caractères physiologiques et nutritionnels très proches des souches orange opaques de la classe I (tableau IV); il en résulte qu'en général, les tests présentés sur le tableau I permettent également de séparer les souches non pigmentées opaques des classes l et
IV. Contrairement aux souches de la classe IV, la classe I forme un groupe assez homogène; toutefois, nous avons distingué 3 sous-classes : 11 (12 souches), I2 ( 8 souches) et I3 ( 2 souches) avec respectivement des distances d'agrégation interne de $0,143,0,146$ et 0,135 . L'utilisation de 8 substrats comme seule source de carbone et d'énergie permet de séparer les souches de la classe I en ces 3 sousclasses (tableau V).

Comme dans le cas des bactéries corynéformes orange opaques, on observe (fig 2) que certains types semblent se rencontrer préférentiellement à certains stades de l'affinage des fromages : c'est le cas par exemple de la classe 12 en fin d'affinage, 
Tableau IV. Comparaison des caractéristiques des souches orange opaques et non pigmentées opaques isolées de la morge du Comté (Pourcentage de réponses positives).

Comparison of characters of the opaque orange and the non pigmented strains of coryneform bacteria isolated from the Comté cheese smear (Percentage of positive strains).

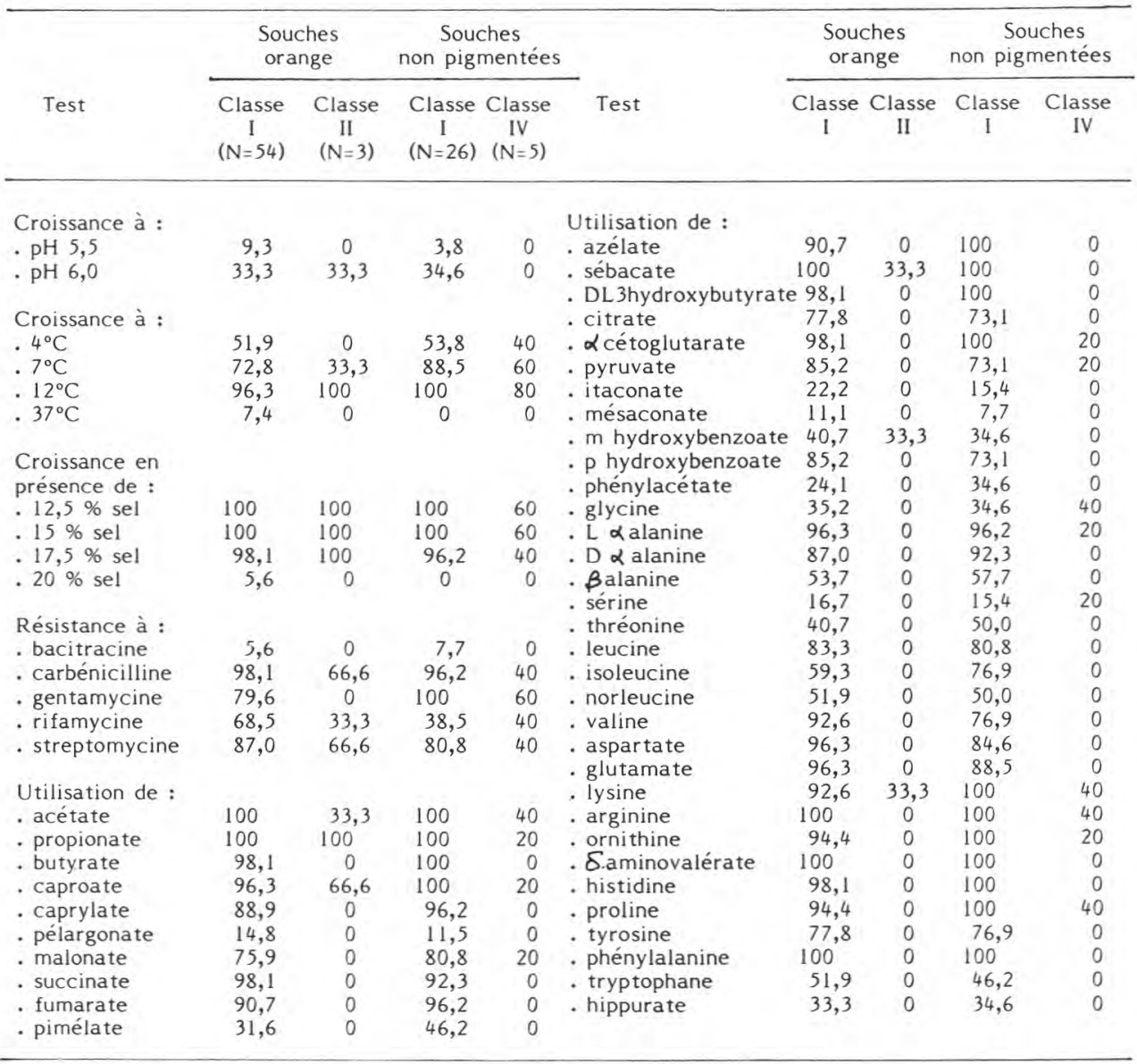

et de la classe 13 en début d'affinage. Cependant, compte tenu du petit nombre de souches par classe, la différence de répartition n'est probablement pas significative.

\section{Colonies translucides}

Sur la figure 2, on note 2 groupes de souches donnant des colonies translu- 
Tableau V. Caractères distinctifs des souches de bactéries corynéformes non pigmentées opaques appartenant aux 3 différentes sous-classes de la classe I (Pourcentage de réponses positives). Biochemical characteristics of opaque non pigmented strains of coryneform bacteria belonging to the 3 subclusters of the cluster I (Percentage of positive strains).

\begin{tabular}{lccc}
\hline \multicolumn{1}{c}{ Test } & Classe I1 & Classe I2 & Classe 13 \\
& & & \\
\hline & & & \\
Utilisation de : & & & \\
- citrate & 91,7 & 87,5 & 0 \\
Pyruvate & 100 & 12,5 & 100 \\
m-hydroxybenzoate & 0 & 87,5 & 50 \\
glycine & 8,9 & 00 & 0 \\
- -alanine & 91,1 & 100 & 0 \\
isoleucine & 83,3 & 100 & 0 \\
aspartate & 91,1 & 87,5 & 0 \\
& 83,3 & & \\
\hline & & 8 & 2 \\
Nyrosine & 12 & 0,146 & 0,135 \\
\hline
\end{tabular}

cides, reliées à la distance de 0,480 : la classe II (18 souches) et la classe III (5 souches), avec respectivement des niveaux d'agrégation interne de 0,333 et 0,348 . Malgré ces niveaux assez élevés de similitude entre souches, nous avons distingué 3 groupes de souches à l'intérieur de la classe II contre 2 groupes dans la classe III. Le tableau VI présente les principaux tests permettant de différencier les bactéries des 2 classes et, à l'intérieur de chaque classe, celles des diverses sous-classes.

Conformément à ce qui a été noté pour les autres groupes pigmentaires, chaque type mis en évidence paraît spécifique d'un stade de l'affinage des fromages : les types II1 et III2 sont rencontrés préférentiellement en début d'affinage tandis que les types II2, II3 et III1 le sont en fin d'affinage. Par contre, aucun type ne semble avoir été isolé plus fréquemment dans l'une ou l'autre des fromageries.

\section{DISCUSSION ET CONCLUSIONS}

D'une façon générale, les résultats de notre étude soulignent une assez grande variabilité des caractères phénotypiques des souches de bactéries corynéformes de la morge du Gruyère de Comté. Toutefois, le groupe des souches orange semble constituer un ensemble homogène, puisque 54 des 59 souches de la collection présentent près de $80 \%$ de similitudes entre elles. La comparaison de ces souches avec celles de bactéries corynéformes décrites dans les études précédentes est souvent difficile, soit en raison du faible nombre de critères communs et ou de souches étudiées, soit en raison de l'origine différente des souches. Cependant, outre la production d'un pigment orange sensible à la soude (Jones et al, 1973), les souches orange isolées du Comté présentent le plus souvent des propriétés analogues à celles de Brevibacte- 
Tableau VI. Caractères distinctifs des souches de bactéries corynéformes non pigmentées translucides appartenant aux différentes classes (Pourcentage de réponses positives).

Characteristics of translucent strains of coryneform bacteria belonging to the different subclusters (percentage of positive strains).

\begin{tabular}{|c|c|c|c|c|c|c|c|}
\hline \multirow{2}{*}{ Test } & \multicolumn{4}{|c|}{ Classe II } & \multicolumn{3}{|c|}{ Classe III } \\
\hline & III & 112 & II 3 & Total & IIII & 1112 & Total \\
\hline & & & & 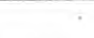 & & & \\
\hline Culture à $\mathrm{pH} 6,0$ & 100 & 0 & 0 & 44,4 . & 50 & 0 & 20 \\
\hline $\begin{array}{l}\text { Utilisation de : } \\
\text { - acétate } \\
\text { - pélargonate } \\
\text { - DL } 3 \text { hydroxybutyrate } \\
\text {. citrate } \\
\text { - dcétoglutarate } \\
\text { - pyruvate } \\
\text { - phénylacétate } \\
\text { - glycine } \\
\text { - L- } \alpha \text {-alanine } \\
\text { - D- } \alpha \text {-alanine } \\
\text { - isoleucine } \\
\text { - valine } \\
\text { - glutamate } \\
\text { - Saminovalérate } \\
\text { - histidine } \\
\text { - phénylalanine } \\
\text {. tryptophane }\end{array}$ & $\begin{array}{c}100 \\
100 \\
100 \\
100 \\
12,5 \\
100 \\
100 \\
0 \\
0 \\
0 \\
0 \\
0 \\
100 \\
87,5 \\
0 \\
0 \\
0\end{array}$ & $\begin{array}{c}100 \\
0 \\
100 \\
0 \\
16,7 \\
83,3 \\
0 \\
0 \\
0 \\
0 \\
0 \\
0 \\
66,6 \\
0 \\
0 \\
0 \\
0\end{array}$ & $\begin{array}{r}100 \\
0 \\
100 \\
0 \\
100 \\
0 \\
0 \\
0 \\
33,3 \\
0 \\
100 \\
100 \\
0 \\
0 \\
0 \\
0 \\
100\end{array}$ & $\begin{array}{c}100 \\
44,4 \\
100 \\
44,4 \\
33,3 \\
72,2 \\
44,4 \\
0 \\
5,6 \\
0 \\
16,7 \\
22,2 \\
66,6 \\
38,9 \\
0 \\
0 \\
16,7\end{array}$ & $\begin{array}{r}0 \\
0 \\
0 \\
50 \\
100 \\
0 \\
0 \\
100 \\
100 \\
0 \\
0 \\
100 \\
0 \\
100 \\
100 \\
100 \\
0\end{array}$ & $\begin{array}{c}0 \\
33,3 \\
0 \\
0 \\
0 \\
100 \\
0 \\
0 \\
100 \\
100 \\
0 \\
0 \\
100 \\
0 \\
100 \\
100 \\
0\end{array}$ & $\begin{array}{r}0 \\
20 \\
0 \\
20 \\
40 \\
60 \\
0 \\
40 \\
100 \\
60 \\
0 \\
40 \\
60 \\
40 \\
100 \\
100 \\
0\end{array}$ \\
\hline Nombre de souches & 8 & 6 & 3 & 18 & 2 & 3 & 5 \\
\hline Niveau d'agrégation & 0,231 & 0,313 & 0,250 & 0,333 & 0,318 & 0,105 & 0,348 \\
\hline
\end{tabular}

rium linens décrites par Mulder et al (1966), Bousfield (1972), Yamada et Komagata (1972), Crombach (1974b) ou Sharpe et al (1977). On notera cependant quelques divergences quant à l'utilisation du citrate et du pyruvate (Bousfield, 1972; Yamada et Komagata, 1972), du pimélate et de I'hippurate (Yamada et Komagata, 1972). Certaines de ces divergences sont sans doute dues aux souches elles-même, mais elles peuvent également être liées à un certain manque de reproductibilité des techniques (Sneath et Johnson, 1972). Par ailleurs, les souches orange isolées du Comté présentent, sur la base d'un nombre assez large de critères, des pro- priétés identiques aux bactéries corynéformes apparentées à $B$ linens isolées de divers fromages (Seiler, 1986).

L'homogénéité sur le plan phénotypique des souches corynéformes orange du fromage a déjà été notée (Crombach, 1974b; Zadi, 1981; Seiler, 1986). II n'est cependant pas certain que cette homogénéité soit une conséquence d'une homologie des souches sur le plan génomique. En effet, Fiedler et al (1981) ont conclu à l'existence de deux espèces parmi les souches décrites comme Brevibacterium linens, grâce à des études d'hybridation de I'ADN; en revanche, l'étude de taxonomie numérique de Seiler (1983) n'a pas permis 
une séparation des souches appartenant à ces deux groupes d'homologie de l'ADN, sur la base de critères nutritionnels. Malgré cette similitude entre souches, nous avons cependant pu distinguer plusieurs types et à l'inverse de Seiler (1986), nous avons isolé une dizaine de tests qui nous permettent d'identifier les souches appartenant à chacun des groupes mis en évidence. Les observations divergentes de Seiler (1986) peuvent avoir plusieurs origines : l'utilisation d'un programme de classification des souches différent, un assez grand nombre de substrats testés différents ou encore le choix des coupures au niveau de l'arbre.

Contrairement à ce qui a généralement été observé dans les études précédentes (Mulder et al, 1966; Sharpe et al, 1977; Zadi, 1981; Seiler, 1986), une forte proportion des souches non pigmentées opaques, isolées de la morge du Gruyère de Comté possède des propriétés physiologiques et nutritionnelles analogues aux souches apparentées à Brevibacterium linens. Par contre, elles se distinguent des souches non pigmentées isolées du fromage et classées comme Brevibacterium casei, en particulier par leur température optimum de croissance (Sharpe et al, 1977; Collins et al, 1983). En outre, elles possèdent certaines propriétés des souches non pigmentées décrites par Mulder et al (1966) et classées plus tard comme Caseobacter polymorphus, en particulier la température de croissance et la tolérance au chlorure de sodium; elles se distinguent cependant de cette espèce par un assez grand nombre de caractères nutritionnels (Crombach, 1974b et 1978; Seiler, 1983 et 1986).

Dans le cas des bactéries orange et des bactéries non pigmentées opaques, nous avons mis en évidence un petit nombre de souches caractérisées par leur incapacité à utiliser la plupart des substrats testés.
Ces souches semblent former des groupes peu homogènes, mais cette hétérogénéité est en partie liée au mode de calcul des distances entre souches. En effet, comme il ne tient compte que des réponses positives, la distance entre ces souches n'a été calculée que sur la base d'une vingtaine de tests sur les 60 de l'étude; une divergence entre deux souches sur un petit nombre de critères se traduit alors par une distance élevée.

Les souches non pigmentées translucides constituent un groupe assez hétérogène qui possède des caractéristiques physiologiques et nutritionnelles assez différentes de celles des deux autres groupes pigmentaires. En particulier, aucune d'entre elles n'est capable de croître à 4 et $7^{\circ} \mathrm{C}$, et environ $20 \%$ d'entre elles à $12^{\circ} \mathrm{C}$; par contre, elles semblent posséder une plus grande tolérance à l'acidité que les souches opaques. En outre, elles sont en général caractérisées par l'incapacité à utiliser un grand nombre des acides aminés testés et elles se distinguent des souches non pigmentées des fromages décrites par Mulder et al (1966), Crombach (1974b), Sharpe et al (1977) ou Seiler (1986). En particulier, sur les 17 critères pour lesquels a eu lieu la comparaison, elles se différencient des souches translucides isolées de divers fromages par Seiler (1986) par 5 à 8 critères. Nous avons cependant noté que les souches de la classe 11 présentent des caractéristiques nutritionnelles semblables à celles décrites par Seiler (1983, 1986) comme Caseobacter polymorphus.

Pour éclaircir la position taxonomique des souches de bactéries corynéformes de la morge du Gruyère de Comté, une étude complète est nécessaire, avec, par exemple, analyse du peptidoglycane de la paroi (Schleifer et Kandler, 1972), du pourcentage d'homologie de l'ADN avec les souches type (Crombach, 1974a; Fiedler 
et al, 1981) ou de la composition en acides gras (Minnikin et al, 1978). Cependant, ce n'était pas le but de notre étude qui était essentiellement de classer les souches en différents types sur la base de critères simples applicables sur un grand nombre de souches. Grâce à l'étude entreprise, nous disposons désormais de ces critères qui devraient nous permettre d'effectuer une étude plus large de la flore de la morge : variabilité en fonction des conditions d'affinage (affinage basse température, fromages hyposodés...), évolution des divers types au cours de l'affinage, flore d'autres fromages emmorgés (Beaufort, par exemple). Les résultats de notre étude nous permettent déjà de faire quelques observations dans ce sens : il ne semble pas exister de spécificité des souches selon l'origine des fromages, et l'existence d'un réservoir unique ou d'une forte pression de sélection sur une flore initiale très complexe à la surface des fromages en est peut-être à l'origine. Cependant, ces observations demandent à être confirmées car les techniques d'affinage conduites chez les différents fromagers et affineurs de l'étude sont relativement proches. Par contre, alors que les nombres de bactéries de chaque groupe pigmentaire se stabilisent à partir de 8 ou 15 jours d'affinage (Piton, 1988), des successions de flores semblent exister à l'intérieur de chaque groupe au cours de l'affinage; ainsi, certains des types mis en évidence sont rencontrés plus fréquemment en début ou en fin d'affinage, mais une étude ultérieure dans un plus grand nombre de fromageries sera nécessaire pour le confirmer.

Sur le plan écologique, les bactéries corynéformes du Gruyère de Comté tolèrent le plus souvent des concentrations très élevées en chlorure de sodium, conformément à ce qui a été observé pour d'autres fromages (Kelly et Marquardt, 1939; Mulder et al, 1966; Sharpe et al, 1976 et 1977; Crombach, 1974b). D'autre part, bien qu'elles croissent à des $\mathrm{pH}$ très élevés, la plupart d'entre elles ne peuvent se développer à pH 6 en absence de chlorure de sodium; de même, leur croissance semble faible à des températures inférieures à $10^{\circ} \mathrm{C}$. Cependant, leur tolérance au $\mathrm{pH}$ et aux températures extrêmes est probablement différente à la surface du Comté où les concentrations en sel peuvent atteindre $12-13 \%$ juste après le salage (Accolas et al, 1978); Mulder et al (1966) ont montré que la présence de sel dans le milieu modifiait la croissance de $B$ linens aux $\mathrm{pH}$ acides et aux températures élevées.

La présence à un niveau élevé de bactéries identifiables à $B$ linens à la surface du Gruyère de Comté est particulièrement intéressante. En effet, il a été montré qu'elles jouent un rôle dans la protéolyse de divers fromages en libérant des acides aminés libres (Ades et Cone, 1969; Tokita et Hosono, 1972), la plupart des souches pouvant libérer de l'ammoniac (EI Erian, 1972). Ces souches produisent également de nombreux composés carbonylés volatils à partir de la caséine (Hosono et Tokita, 1969), des composés soufrés volatils (Grill et al, 1967; Parliment et al, 1982; Hemme et Richard, 1986) ou des phénols (Parliment et al, 1982). Or, la plupart de ces composés ont été mis en évidence dans la zone corticale des fromages de Comté et de Beaufort (Dumont et Adda, 1978; Dumont et al, 1981). D'autres types de bactéries corynéformes isolés à des niveaux semblables à la surface du Comté peuvent également participer à la protéolyse et à la production de composés d'arômes, en particulier les souches non pigmentées opaques qui possèdent des caractères phénotypiques très proches de celles apparentées à $B$ linens. 


\section{RÉFÉRENCES}

Accolas JP, Melcion D, Vassal L (1978) Étude de la flore superficielle des fromages de Gruyère et de Beaufort. $20^{\circ}$ Cong Int Lait, Paris, 773-774

Ades GL, Cone JF (1969) Proteolytic activity of Brevibacterium linens during ripening of Trappist-type cheese. J Dairy Sci 52, 957961

Benzecri JP (1975) L'analyse des données. I. La taxonomie. Dunod, Paris

Bousfield IJ (1972) A taxonomic study of some coryneform bacteria. J Gen Microbiol 71, 441-455

Collins MD, Farrow JAE, Goodfellow M, Minnikin DE (1983) Brevibacterium casei sp nov and Brevibacterium epidermitis sp nov. Syst Appl Microbiol 4, 388-395

Crombach WHJ (1974a) Relationships among coryneform bacteria from soil, cheese and sea fish. Antonie Van Leeuwenhoek J Microbiol 40, 347-359

Crombach WHJ (1974b) Morphology and physiology of coryneform bacteria. Antonie Van Leeuwenhoek J Microbiol 40, 361-376

Crombach WHJ (1978) Caseobacter polymorphus gen nov, sp nov, a coryneform bacterium from cheese. Int $J$ Syst Bacteriol 28 , 354-366

Dumont JP, Adda J (1978) Occurrence of sesquiterpenes in mountain cheese volatiles. $J$ Agric Food Chem 26, 364-367

Dumont JP, Adda J, Rousseaux P (1981) Exemple de variation de l'arôme à l'intérieur d'un même type de fromage : le Comté. Lebensm Wiss Technol 14, 198-202

El Erian AFM (1972) Bacteriological studies on Limburger cheese. Neth Milk Dairy J 26, 113116

Fiedler F, Schaffler MJ, Stackebrandt E (1981) Biochemical and nucleic acid hybridisation studies on Brevibacterium linens and related strains. Arch Microbiol 129, 85-93

Grill H, Patton S, Cone JF (1967) Degradation of ${ }^{35} \mathrm{~S}$-methionine to methyl mercaptan in surface ripened cheese. J Agric Food Chem $15,392-393$

Hemme D, Bouillanne C, Metro F, Desmazeaud MJ (1982) Microbial catabolism of aminoa- cids during cheese ripening. Sci Aliments 2, 113-123

Hemme D, Richard J (1986) Utilization of Lmethionine and production of methanethiol by bacteria isolated from raw milk Camembert cheese. Lait 66, 135-142

Hosono A, Tokita F (1969) Studies on the decarboxylation of aminoacids by Brevibacterium linens. Jpn J Zootech Sci 40, 544-550

Jones D (1975) A numerical taxonomic study of coryneform and related bacteria. J Gen Microbiol 87, 52-96

Jones D, Watkins J, Erickson SK (1973) Taxonomically significant colour changes in Brevibacterium linens probably associated with a carotenoid-like pigment. J Gen Microbiol 77, $145-150$

Kelly CD, Marquardt JC (1939) The influence of hydrogen ion concentration and salt on the surface flora of Limburger cheese. I Dairy Sci 22, 309-320

Kikuchi T (1966) Observation on Camembert cheese ripening. 17th Int Dairy Congr D, 313319

Lenoir J (1963) La flore microbienne du camembert et son évolution au cours de la maturation. Lait 43, 262-270

Lenoir J, Auberger B (1966) Contribution à l'étude de la flore microbienne du fromage de type camembert. 17th Int Dairy Congr D2, 595-602

Minnikin DE, Goodfellow M, Collins MD (1978) Lipid composition in the classification and identification of coryneform and related taxa. In: Special Publications of the Society for $\mathrm{Ge}$ neral Microbiology. I. Coryneform bacteria (Bousfield and Callely, eds) Academic Press, London, 85-160

Mourgues R, Vassal L, Auclair J, Mocquot G, Vandeweghe J, Deschamps N (1977) Origine et développement des bactéries coliformes dans les fromages à pâte molle. Lait 57, 131149

Mulder EG, Adamse AD, Antheunisse J, Deinema MH, Woldendorp JW, Zevenhuizen LPTM (1966) The relationship between Brevibacterium linens and bacteria of the genus Arthrobacter. J Appl Bacteriol 29, 44-71

Parliment TH, Kolor MG, Rizzo DJ (1982) Volatile components of Limburger cheese. J Agric Food Chem 30, 1006-1008 
Piton C (1988) Évolution de la flore microbienne de surface du gruyère de Comté au cours de l'affinage. Lait 68, 419-434

Richard J (1984) Évolution de la flore microbienne à la surface des camemberts fabriqués avec du lait cru. Lait 64, 496-520

Richard J, Zadi H (1983) Inventaire de la flore microbienne dominante des camemberts fabriqués avec du lait cru. Lait 63, 25-42

Schleifer KH, Kandler O (1972) Peptidoglycan types of bacterial cell walls and their taxonomic implications. Bacteriol Rev 36, 407-477

Seiler H (1983) Identification key for coryneform bacteria derived by numerical taxonomic studies. J Gen Microbiol 129, 1433-1471

Seiler H (1986) Identification of cheese-smear coryneform bacteria. J Dairy Res 53, 439449

Sharpe ME, Law BA, Phillips BA (1976) Coryneform bacteria producing methane thiol. $J$ Gen Microbiol 94, 430-435

Sharpe ME, Law BA, Phillips SA, Pitcher DG (1977) Methanethiol production by coryneform bacteria : strains from dairy and human skin sources and Brevibacterium linens. $J$ Gen Microbiol 101, 345-349

Sneath PHA, Johnson R (1972) The influence on numerical taxonomic similarities of errors in microbiological tests. J Gen Microbiol 72, 377-392

Sokal RR, Sneath PHA (1963) Principles of numerical taxonomy. Freeman WH \& Co, London

Stanier RY, Palleroni NJ, Doudoroff M (1966) The aerobic Pseudomonas: a taxonomic study. J Gen Microbiol 43, 159-271

Tokita F, Hosono A (1972) Studies on the extracellular protease produced by Brevibacterium linens. I. Production and some properties of the extracellular protease. Jpn J Zootech Sci 43, 39-48

Yamada K, Komagata K (1972) Taxonomic studies on coryneform bacteria. IV. Morphological, cultural, biochemical and physiological characteristics. J Gen Appl Bacteriol 18, 399416

Zadi H (1981) Etude de la flore bactérienne non lactique des Camemberts fabriqués de façon traditionnelle. Thèse, Orsay. 